\title{
La educación universitaria y el proceso de reincorporación, una visibilización necesaria para trascender el enfoque de la armadura vacía*
}

\author{
[Artículos]
}

\author{
Ximena del Pilar Rincón Castellanos ${ }^{* *}$ \\ Jenniffer Pinilla León $n^{* * *}$
}

Recibido: 10 febrero de 2021

Aceptado: 6 de mayo de 2021

Citar como:

Rincón Castellanos, X. d. P. y Pinilla León, J. (2021). La educación universitaria y el proceso de reincorporación, una visibilización necesaria para trascender el enfoque de la armadura vacía. Revista IUSTA, (55).

https://doi.org/10.15332/25005286.6853

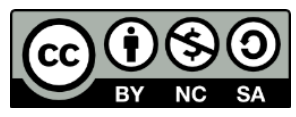

\footnotetext{
* Artículo derivado del proyecto de investigación "La educación como derecho de la población desmovilizada en la política pública para la paz y su compatibilidad con los estándares de calidad de la educación superior en el marco de la autonomía universitaria" (código INV DER 2555), financiado por la Vicerrectoría de Investigaciones de la Universidad Militar Nueva Granada, Colombia, vigencia 2018.

** Abogada de la Universidad Pedagógica y Tecnológica de Colombia (UPTC) y magíster en Política Social de la Pontificia Universidad Javeriana. Auxiliar Judicial Grado 1 Corte Constitucional de Colombia. Correo electrónico: xilarpc19@yahoo.es; ORCID: https://orcid.org/0000-0002-1250-6848

${ }_{* * *}$ Abogada de la Pontificia Universidad Javeriana y magíster en Derecho de la Universidad Externado de Colombia. Docente de la Universidad Militar Nueva Granada y de la Pontificia Universidad Javeriana. Correo electrónico:

jenniffer.pinilla@unimilitar.edu.co; ORCID: https://orcid.org/0000-0002-3152-0733
} 


\section{Resumen}

Colombia ha asumido desde hace varios años procesos de reintegración y reincorporación de personas vinculadas a grupos al margen de la ley, orientados a consolidar una paz estable y duradera. Los esfuerzos institucionales, colectivos e individuales han buscado brindar herramientas para asumir las diversas dimensiones en las que se ejerce la ciudadanía en un escenario de posconflicto. La educación es una de estas dimensiones, concebida como aquella de la que depende el éxito de muchos de los esfuerzos del proceso. Pese a que se han diseñado importantes propuestas para los excombatientes, que van desde ciclos de educación básica y media hasta formación para el empleo, se ha privilegiado la oferta técnica y tecnológica, que, si bien se concibe como educación superior, no se ha articulado ni complementado con la oferta universitaria, que a su vez se encuentra sujeta a lineamientos de calidad que implican retos que continúan pendientes de resolver.

Palabras clave: calidad educativa, educación superior, inclusión social, reincorporación.

\section{University education and the reincorporation process, a necessary visibilization to transcend the empty armor approach}

\section{Abstract}

For several years, Colombia has been undertaking the processes of reintegration and reincorporation of people belonging to illegal groups, aimed at consolidating a stable and lasting peace. Institutional, collective and individual efforts have sought to provide tools to assume the different dimensions in which citizenship is exercised in a post-conflict scenario. Education is one of these dimensions, understood as that on which the success of many of the efforts of the process depends. Although important proposals have been designed for ex-combatants, ranging from basic and secondary education cycles to job training, the technical 
and technological offer has been favored, which, although conceived as higher education, has not been articulated or complemented with the university offer, which in turn is subject to quality guidelines that imply challenges that are still pending to be solved.

Keywords: educational quality, higher education, social inclusion, reincorporation.

\section{O ensino universitário e o processo de reincorporação, uma visibilização necessária para transcender a abordagem da armadura vazia}

\section{Resumo}

A Colômbia vem assumindo, há vários anos, processos de reintegração e reincorporação de pessoas vinculadas a grupos à margem da lei, orientados a consolidar uma paz estável e duradoura. Os esforços institucionais, coletivos e individuais têm buscado oferecer ferramentas para assumir as diversas dimensões nas quais a cidadania é exercida em um cenário de pós-conflito. A educação é uma dessas dimensões, concebida como aquela da qual o sucesso de muitos esforços do processo depende; embora importantes propostas para os ex-combatentes tenham sido desenhadas, que vão desde ciclos de educação básica e média até formação para o emprego, a oferta técnica e tecnológica tem sido privilegiada que, apesar de conceber-se como educação superior, não tem sido articulado nem complementado com a oferta universitária, que, por sua vez, se encontra sujeita a lineamentos de qualidade que implicam desafios que continuam pendentes.

Palavras-chave: qualidade educativa, ensino superior, inclusão social, reincorporação.

\section{Introducción}

Italo Calvino, en su libro El caballero inexistente, crea un personaje llamado Agilulfo, quien se caracteriza por no tener un cuerpo. Es un 
caballero que habita las tierras francesas gobernadas por Carlomagno a través de una armadura que está vacía. Posee una profunda destreza en su oficio de paladín, conoce todos los procedimientos, las historias de las batallas y los detalles de las hazañas del emperador, es un perfeccionista y se ocupa con extremo cuidado de cada una de sus tareas. Su armadura blanca se distingue de las de los demás caballeros y es reconocido por el refinado control de la espada, en conclusión, es un perfecto técnico que domina el saber con bastante suficiencia.

En la trama de la historia son visibles los contrastes entre las respuestas de Agilulfo a los desafíos vitales de su época y las de los demás caballeros, quienes sí tienen un cuerpo y están sujetos a ello, tanto por lo material, evidenciado en la necesidad de dormir o comer, como por lo inmaterial, ligado a las emociones y las necesidades de reconocimiento, iel honor! Agilulfo emprende una ruta para encontrar a alguien que le sirva de testigo y demostrar que el rescate que realizó hace varios años y que le otorgó el título de nobleza que le permitió ser caballero realmente ocurrió en ciertas circunstancias, pero no lo hace motivado por sus emociones, pues no están presentes dado que no tiene cuerpo, sino porque es el procedimiento estipulado en el manual de buen caballero, a saber, disponer los medios necesarios para establecer la precisión fáctica de hechos del pasado. Una operación técnica con ausencia de emociones, valoraciones o consideraciones éticas o interpersonales.

La metáfora de la armadura vacía es un recurso tan fantástico como didáctico para reflexionar en torno a la condición humana y cuál debería ser el papel del sistema educativo en un contexto tan particular y exigente como la reincorporación de actores armados a la vida civil. Los seres humanos no somos armaduras vacías que requieren únicamente el dominio de una técnica para sobrevivir, somos mucho más complejos y esa 
pericia es solo una pequeña porción del propósito que debería cumplir la educación, que si bien puede priorizar en una de estas pretensiones, no puede desconocer ni desatender las expectativas de quienes desean acceder a otros escenarios de formación.

En un contexto global que cada vez jalona con más fuerza la automatización de los oficios y por eso los trabajadores van siendo reemplazados por robots, son cada vez más valiosos los saberes humanos que resuelven la convivencia y permiten que las instituciones de una sociedad funcionen; en palabras de Martha Nussbaum (2010), la democracia necesita de las humanidades.

Por consiguiente, en un proceso de reincorporación, la oferta educativa no puede considerarse plenamente satisfecha cuando privilegia exclusivamente la educación técnica. Si los excombatientes y los ciudadanos fueran armaduras vacías, no aspirarían a la reincorporación y, por tanto, entendiendo la importancia del proceso, la educación debe ser contemplada desde una perspectiva más compleja, incluyente y al alcance de aquellos que buscan en ella alternativas que vayan más allá del perfeccionamiento de la técnica o el oficio (Moreno y Álvarez, 2017). Adicionalmente, es claro que las instituciones educativas en general y las instituciones de educación superior (IES) en particular deben cumplir con una serie de parámetros y lineamientos que fijan el estándar de calidad de los programas, además, cuentan con la posibilidad de fijar sus propias políticas en el marco de la autonomía universitaria. Estas dos variables cumplen una función importante en las propuestas que las IES pueden formular para acoger a la población en proceso de reincorporación. Así, la pregunta se orienta a identificar, en el marco de las necesidades y aspiraciones de la población en proceso de reincorporación, cuáles son las propuestas que se han diseñado en la política pública para la paz para y 
por las IES, y cómo es su articulación con los estándares de calidad establecidos por el Ministerio de Educación Nacional (MEN), en el marco de la autonomía universitaria.

Estas preguntas orientan las reflexiones del presente artículo que, en un primer momento, se dedica a clarificar algunos conceptos a partir de cifras oficiales, para abordar posteriormente las barreras existentes para la población en proceso de reincorporación e identificar los retos que deben ser asumidos para responder a una aspiración legítima de acceso a escenarios universitarios, como parte del proceso de reincorporación, en el marco de los lineamientos del MEN.

La investigación que dio lugar a este artículo se desarrolló siguiendo una metodología de corte cualitativo, descriptivo e interpretativo, enfocada en el análisis teórico de fuentes oficiales, tales como el MEN, el Consejo Nacional de Acreditación (CNA) y la Agencia para la Reincorporación y la Normalización (ARN). También se consultaron las páginas web oficiales de universidades, tanto públicas como privadas. El estudio se limitó a la información contenida en bases de datos y páginas web, en un periodo aproximado de 24 meses, entre febrero de 2018 y junio de 2020.

\section{$D^{2}$ y $\mathbf{R}^{2}$ : ¿cuál es el contexto para nuestra reflexión?}

La precisión sobre el significado de varias palabras que vienen siendo usadas en los últimos años no es solo una urgencia semántica, sino una referencia pertinente tanto para cualificar el debate (Aristizábal, 2019), como para entender el alcance profundo de los procesos que están en juego en Colombia con las decisiones presentes y futuras en relación con desarme, desmovilización, reintegración y reincorporación (DDR).

DDR es la expresión más usada para referirse a un proceso compuesto por cuatro momentos, el nombre de los dos primeros comienza con la letra $\mathrm{D}$, 
mientras que el tercero y el cuarto con la R, de ahí que en el presente artículo proponemos representarlo como $\mathrm{D}^{2}$ y $\mathrm{R}^{2}$.

El desarme es entendido como la dejación de armas y la recogida de las armas, por lo general ligeras, en conflictos internos o guerras civiles, dentro de las zonas de conflicto definidas y en tiempos determinados y regularmente a partir de concentraciones para el efecto, de forma que se determinan o convienen términos para concentrar o confiscar, almacenar y destruir o inutilizar tal armamento. (CNMH, 2015, p. 21)

Doris Salcedo, reconocida en Colombia por ser una artista que ha realizado varias obras en respuesta a actos de violencia como la toma del Palacio de Justicia, lideró un grupo de mujeres víctimas de agresiones sexuales en el marco del conflicto armado y juntas construyeron el antimonumento denominado Fragmentos, que utilizó como materia prima las armas entregadas por los excombatientes de las Fuerzas Armadas Revolucionarias de Colombia-Ejército del Pueblo (Farc-EP) ${ }^{1}$. La desmovilización de actores armados es un proceso que tiene efectos a nivel individual y estructural. Este último significa el quiebre de la organización armada y su "desmantelamiento", y en lo personal implica “liberar a los combatientes de un estado de movilización”. En este aspecto radica el fundamento de la existencia de la justicia transicional (Ortega y García, 2019). Los beneficios penales y sociales son la motivación para que el grupo armado desista de sus acciones bélicas, de manera que la justicia transicional no está concebida para ser una herramienta de guerra del Estado contra su enemigo en el campo de batalla, sino como una estrategia de pacificación. En esa lógica, la política que incentiva a través de

\footnotetext{
${ }^{1}$ Detalles de la obra pueden ser consultados en varios artículos. Uno de ellos está disponible en RevistaArcadia.com (2018).
} 
beneficios penales la desmovilización individual, con el propósito de obtener información útil para debilitar al enemigo, aviva el conflicto. La justicia transicional tiene sentido y es justificable ante la sociedad y la comunidad internacional porque permite que el grupo armado desaparezca y, en consecuencia, la guerra se debilite (CNMH, 2015, p. 21). El uso indebido de las medidas transicionales como estrategia para derrotar al "enemigo" va en contravía de los estándares internacionales de derechos humanos y derecho internacional humanitario.

Después de la desmovilización, la reinserción es la "llegada de las personas excombatientes a su lugar de origen o destino para vincularse en condiciones de legalidad" (CNMH, 2015, p. 21; énfasis añadido). Esta fase implica la asistencia inmediata con ropa, alimentos, refugio y servicio médico. Finalmente, la reincorporación es el proceso más extenso y que de manera profunda desafía a la sociedad civil y a otras instituciones del Estado que hasta el momento habían sido observadoras. Este momento apunta a que los(as) excombatientes se vinculen de manera efectiva a la vida civil y las variables de éxito van desde garantías de sostenibilidad de las condiciones de vida de estas personas hasta la "superación de las causas de conflictividad y la recuperación social y económica de las comunidades receptoras” (CNMH, 2015, p. 22; énfasis añadido).

En este contexto, para efectos del presente artículo, es necesario distinguir entre la reintegración y la reincorporación, dado que con la suscripción de los acuerdos de paz de La Habana, estos conceptos fueron redimensionados y redefinidos.

Según la ARN (s. f.), la reintegración se define como:

una oferta de seis años y medio que el Estado colombiano, a través de la gestión que realiza la ARN, les ofrece a las personas desmovilizadas de 
los Grupos Armados Organizados al Margen de la Ley (GAOML), que no han cometido delitos de lesa humanidad, y que quieren reintegrarse a la vida social y económica.

Tal como lo explica el informe de la ARN (2020) en materia de reintegración, la Agencia avanza con dos procesos: 1) reintegración regular: comprende a la persona en proceso de reintegración, así como a su familia, en ocho dimensiones; y 2) la reintegración especial, que aborda el proceso de las personas postuladas a la Ley 975 de 2005 que recobran su libertad efectiva, con acompañamiento psicosocial, formación académica, entre otros, en procura de su sostenibilidad en la legalidad y el cumplimiento del compromiso con las víctimas.

Por su parte, el Plan Nacional de Desarrollo (PND) 2014-2018 señala que la reintegración implica para quienes deciden dejar las armas una posibilidad de vivir dignamente, en la legalidad y contribuir a la paz, la seguridad y la convivencia. Este propósito implica generar mecanismos con la debida articulación de las entidades públicas, que permitan superar la estigmatización, promover la estabilidad jurídica, el ejercicio autónomo de la ciudadanía, así como la existencia de mecanismos para proteger la vida, la libertad, la integridad y la seguridad de quienes han depuesto las armas (DNP, 2015).

Tal como se ha establecido, al proceso de reintegración pueden acceder las personas que hayan sido acreditadas como desmovilizadas por el Comité Operativo para la Dejación de Armas (CODA) o la oficina del Alto Comisionado para la Paz, después del 24 de enero de 2003, entre quienes pueden estar exintegrantes de las Frac-EP, del Ejército de Liberación Nacional (ELN), del Ejército Popular de Liberación (EPL) y de las Autodefensas Unidas de Colombia (AUC) (ARN, 2020a). 
En contraste, la reincorporación se concibe como: "un proceso de estabilización socioeconómica de los excombatientes que entregaron sus armas, en el marco de la firma del Acuerdo Final entre el Estado y las Farc-EP” (ARN, 2020a). Esta definición, resultado de la negociación y definición del Acuerdo Final (DNP, 2018, p. 13), plantea un aspecto singular dada la co-construcción que sus acciones evidencian, al tratarse de estrategias concertadas y definidas de manera conjunta principalmente con el Consejo Nacional de Reincorporación (CNR).

A partir de esta precisión de conceptos, las reflexiones planteadas en este artículo se centran en el desafío de la reincorporación, que va más allá de la reinserción, al proponerse transformar la denominación "excombatiente" por la de "ciudadano", en la forma y en las profundidades de las relaciones sociales. No obstante, muchas de las barreras y retos que se identifican pueden hacerse extensivas a las situaciones que viven las personas en proceso de reintegración, dado que constituyen aspectos que hemos identificado como persistentes en las encuestas y documentos institucionales que fueron usados como insumo en la revisión documental realizada para desarrollar el presente artículo.

\section{Algunas cifras del proceso}

Previo a la firma del acuerdo de paz con las Farc-EP ya se realizaban esfuerzos por avanzar en procesos de reintegración, dados los intentos de desmovilización previa adelantados con otros grupos al margen de la ley. En este sentido, es posible encontrar algunos textos que no solo avocan el estudio del impacto efectivo de la educación en el proceso de reintegración, sino que abordan las estrategias concretas que a nivel gubernamental han sido emprendidas, así como las que se orientan a 
identificar los aportes que las IES han efectuado para contribuir con estos propósitos.

El Observatorio de Desarme Desmovilización y Reintegración (ODDR) de la Universidad Nacional de Colombia es un ejemplo de este ejercicio de análisis. En el informe presentado en el año 2013, el Observatorio señaló que 631 excombatientes en proceso de reintegración realizaron estudios a nivel profesional, durante el periodo 2007-2013, distribuidos en IES ubicadas en Antioquia, Cesar, Meta y Distrito Capital, para un total de 25 departamentos incluidos y 145 programas curriculares.

Asimismo, el ODDR estableció que las carreras de derecho, administración de empresas, ingeniería de sistemas y contaduría pública fueron las opciones más recurrentes que concentraron el mayor número de población en proceso de reintegración; asimismo, los estudiantes estaban adscritos a 114 IES, de las cuales el $76 \%$ pertenecía al sector privado (ODDR, 2013, pp. 27-29).

Por otra parte, con ocasión de la reorientación de los procesos ocasionada por la firma del acuerdo de paz y los compromisos que se derivaron de este, se acopian los datos que dan cuenta de los avances en el proceso de implementación, considerando las metas fijadas en el PND 2014-2018 y el monitoreo necesario de las estrategias orientadas al fortalecimiento de la Política de Reintegración Social y Económica (PRSE).

La ARN y el Conpes 3931 registraron que 52234 personas ingresaron al proceso de reintegración desde noviembre de 2003 hasta junio de 2018, de las cuales 24880 han culminado de manera exitosa el proceso, al superar niveles de alfabetización primaria, secundaria y media. De esta población, el $3 \%$ indicó contar con formación universitaria al momento de ingresar al GAOML. 
Según datos aportados por Fucepaz y el Consejo Noruego para Refugiados, de los 6370 exintegrantes de las Farc-EP, el $67 \%$ de los hombres y el $76 \%$ de las mujeres manifestaron su interés por terminar el bachillerato; por su parte, el 49 \% expresó su interés en acceder a educación superior (DNP, 2018, p. 65). Asimismo, en el censo realizado por el ODDR, el $85 \%$ de las mujeres indicó que la actividad que más les interesa es estudiar, mientras que en el caso de los hombres este interés fue del $69 \%$.

La educación superior hace parte de los beneficios de la reintegración, tal como se verifica en los documentos institucionales, pero es claro que las propuestas y modelos que se proponen deben cumplir con las competencias y estándares de calidad establecidos de manera previa por el Ministerio de Educación Nacional (MEN), entidad a la que le corresponde proferir la normatividad sobre la materia, de acuerdo con las realidades que evidencia la población, teniendo en cuenta, por ejemplo, la necesidad de fortalecer habilidades básicas como la lectoescritura, el reconocimiento de saberes previos y la flexibilidad necesaria para posibilitar el acceso y permanencia en el sistema.

Según el informe de la Secretaría General de la Misión de Verificación de las Naciones Unidas en Colombia (ONU, 2019), un total de 3543 excombatientes (incluidas 914 mujeres) están inscritos en programas de formación para el trabajo a través del Servicio Nacional de Aprendizaje (SENA), además, varias universidades e instituciones de educación superior ofrecen oportunidades similares para cualificar este tipo de oficios y habilidades a través de ofertas como diplomados, entre otras (ONU, 2019, p. 7).

Asimismo, conforme a las cifras recogidas por la ARN, al 30 de abril del año 2020, 1468 personas se encontraban matriculadas en formación académica, 761 en el modelo de formación para adultos (MEFR) y se 
habían otorgado 25 becas de la Universidad Elite y la Organización de Estados Iberoamericanos (OEI), cifra que no se ha modificado desde agosto de 2019, según los reportes publicados por la entidad.

Por lo tanto, el registro estadístico enunciado permite verificar que la aspiración de acceso a la educación superior por parte de la población en proceso de reincorporación constituye una pretensión que, si bien no es mayoritaria dado que depende de diversos factores - muchos de ellos determinados por la priorización de satisfacer las necesidades básicas-, sí constituye un aspecto importante en la agenda del proceso de reincorporación. Sin embargo, es claro que su abordaje implica no solo una perspectiva económica orientada al cubrimiento de los costos de matrícula y condiciones de acceso a estos beneficios, sino una revisión desde los requisitos, oferta de programas, cobertura, acceso, adaptación y permanencia, de una población que tiene una expectativa y una interpretación particulares frente al sistema educativo y que debe avanzar en el ejercicio de su ciudadanía en libertad. Además de satisfacer estas necesidades propias de los excombatientes, el sistema educativo también debe cumplir con unos estándares y requisitos para garantizar su sostenibilidad económica y su posicionamiento académico, lo que constituye un reto pero también una reflexión constante, que a manera de balanza ubica a los dos extremos del binomio, en la necesidad de equilibrar sus expectativas con sus posibilidades. 


\section{La inclusión de excombatientes en la educación superior}

\section{El enfoque de la armadura vacía}

A diferencia del estándar fijado por el Comité de Derechos Económicos, Sociales y Culturales de las Naciones Unidas ${ }^{2}$ en la Observación General n. ${ }^{\circ}$ 13, en la política pública colombiana la educación técnica y tecnológica es considerada parte del nivel de enseñanza superior (Ley 30 de 1992), mientras que para ese órgano es complementaria de la primaria, secundaria y universitaria o superior. Esto significa que en la línea base, por un lado, y en la evaluación de resultados de la política de educación superior, por otro, las cifras de estudiantes admitidos que cursan y se gradúan de instituciones técnicas y tecnológicas engrosan las estadísticas de cobertura, permanencia y egreso de las universidades. En esta misma perspectiva, las IES, el SENA y las demás instituciones que ofrecen programas técnicos y tecnológicos son consideradas universidades.

En ese contexto, el SENA ha sido el oferente principal para asegurar educación superior a la población desmovilizada en Colombia, en conexión con la estrategia de empleabilidad e inserción laboral. Así lo demuestran los estudios del ODDR, en los que se evidencia el convenio entre esa entidad y la entonces Agencia Colombiana para la Reintegración, en el que se pactó que dicha institución otorgaría "hasta el $20 \%$ de los cupos de la oferta trimestral en formación titulada" a favor de la población desmovilizada (ODDR, 2013; énfasis añadido).

Particularmente, el documento Conpes 3931 (DNP, 2018) dispuso que:

\footnotetext{
2 Este es un órgano conformado por expertos de diferentes países y creado por el Consejo Económico Social de las Naciones Unidas, con el objetivo de orientar y supervisar a los Estados parte del Pacto Internacional de Derechos Económicos, Sociales y Culturales, en el cumplimiento de las obligaciones internacionales adquiridas tras la firma y ratificación de este tratado.
} 
La ARN coordinará con las entidades competentes, particularmente el Ministerio del Trabajo, el Servicio Nacional de Aprendizaje (SENA), la Unidad de Servicio Público de Empleo y la UAEOS y los espacios de coordinación como el CNR, el diseño del instrumento de caracterización poblacional que permita orientar a los exintegrantes de las Farc-EP hacia los servicios de gestión y colocación, de emprendimiento y desarrollo empresarial, en contextos urbanos y rurales.

De modo que con el legítimo objetivo de que los excombatientes obtengan un trabajo, se privilegia la oferta de formación para aprender oficios como panadería y mecánica, para los hombres, y belleza, para las mujeres. De modo que el dominio de un oficio es prioritario en la continuidad del proceso educativo, mientras que el acceso a la educación superior, entendida como estudios universitarios, resulta un punto incluido más no priorizado en la agenda.

En este sentido, una primera barrera para la población en proceso de reincorporación que busca formación universitaria, así como para las instituciones llamadas a atender esta aspiración, es que, acogiendo el relato de Agilulfo, se les vea como una armadura vacía cuyo único saber hacer es la guerra y que por ello se considere que su necesidad más urgente en la dimensión educativa es aprender un oficio productivo.

Asimismo, es claro que la medición sobre el alcance de la política en esta materia refleja un avance importante. Sin embargo, no puede ser valorado y asumido de manera escueta si se tiene en cuenta que su medición incluye para su aforo a la enseñanza técnica y tecnológica, pese a que desde el punto de vista normativo y funcional la formación profesional universitaria plantea unas diferencias que no solo reconocen las disposiciones de la reincorporación, sino que además constituyen aspiraciones de quienes se han acogido a estos procesos. 


\section{El enfoque universitas}

El término "universidad" tiene su origen en la palabra latina universĭtas, que se refiere a una comunidad de profesores y académicos, constituida como institución de enseñanza superior, que otorga grados académicos (Navarrete, 2013, p. 5). La universidad ha sido entendida bajo la premisa de la universalidad, como un espacio para la convivencia diversa en la que los distintos grupos humanos pueden encontrarse para pausar el oficio cotidiano y, con tiempo y otros marcos teóricos, ojalá opuestos, reflexionar sobre la sociedad desde los roles de estudiantes, investigadores y docentes. En la universidad la destreza con el oficio es valorada en el contexto social, político y económico, pero no de manera aislada, sino con base en su utilidad concreta.

La Ley 30 de 1992 indica que las universidades son IES, al igual que las instituciones técnicas profesionales y las instituciones universitarias o escuelas tecnológicas (Artículo 16), de manera que las universidades deben, además de contar con el reconocimiento estatal, desempeñar su misión con criterios de universalidad en actividades como: investigación científica o tecnológica, formación académica en profesiones o disciplinas y la producción, desarrollo y transmisión del conocimiento y de la cultura universal y nacional (Artículo 19).

En igual sentido, la normativa precisa que constituye un principio de la educación superior despertar en los educandos un espíritu reflexivo, con miras al logro de una autonomía personal, enmarcado dentro de la libertad de pensamiento y el pluralismo ideológico. La Ley ratifica que la educación superior debe desarrollarse teniendo en cuenta la universalidad de los saberes y la particularidad de las formas culturales existentes en el país (Ley 30 de 1992, art. 4, Colom.). 
Esto se complementa con una garantía reconocida a las IES, a saber, la autonomía universitaria, un principio que fundamenta la educación superior y que según las previsiones del Artículo 30 plantea como aspecto propio de estas instituciones, la búsqueda de la verdad, el ejercicio libre y responsable de la crítica, de la cátedra y del aprendizaje (Ley 30 de 1992, art. 30, Colom.).

En este sentido, es claro que la educación como derecho, enmarcada dentro de esa noción de educación superior, tiene como actor esencial a las IES, que actúan bajo la premisa de promover el pleno desarrollo de los alumnos para garantizar su formación académica y profesional.

El papel esencial que desempeñan las IES como escenarios de educación superior se concreta en el hecho de que son espacios propicios para la construcción de identidades profesionales (Navarrete, 2013, p. 6), para la reflexión y el ejercicio de la ciudadanía, así como para la formulación de propuestas a partir de las iniciativas investigativas desarrolladas bajo una perspectiva plural.

En este sentido, las responsabilidades que les han sido asignadas a las IES evidencian la importancia de su articulación con los procesos de reincorporación, dado que no solo ofrecen a los sujetos habilidades para la subsistencia, sino que además potencian la convivencia, la comprensión de los referentes que hacen parte de su proyecto de vida, promueven la reflexión sobre su entorno y la postura crítica ante su propia realidad. "Para un excombatiente, el hecho de iniciar una carrera profesional compromete una perspectiva de futuro. Además, es la demostración de un ejercicio de ciudadanía y convivencia que, de forma privilegiada, apunta a la sostenibilidad de una apuesta civil” (ODDR, 2013, p. 15).

Hasta el año 2013, el ODDR dio cuenta de los avances normativos, así como de las estrategias institucionales que vinculaban a la educación 
superior con los procesos de reintegración que se adelantaban en ese momento. En efecto, el plexo normativo expedido hasta entonces daba cuenta de una serie de disposiciones orientadas a generar mecanismos para acceder a los beneficios socioeconómicos que permitieran el acceso a la educación superior.

El informe mencionado (ODDR, 2013) destaca la Resolución 549 de 1997 que consagraba la exoneración de la matrícula a excombatientes para cursar programas de formación tecnológica, universitaria y avanzada ofrecida por la Escuela Superior de Administración Pública (ESAP), el Decreto 128 del 23 de enero de 2003 que establecía también posibilidades de financiación parcial para programas de educación superior y la Resolución 513 de 2005 que, si bien reconoce financiación para algunos programas (nivel tecnológico y tecnológico profesional), excluyó de las posibilidades de apoyo a los programas de formación profesional cuya duración fuera de 8 a 10 semestres. Asimismo, se recoge la Política Nacional de Reintegración Social y Económica para personas y grupos armados al margen de la ley, consagrada en el documento Conpes 3554, en la cual se ubica a la educación como eje central de la apuesta por la reintegración. Esta política señala, entre otros aspectos, la importancia de la articulación con la educación básica y media, la financiación y preparación para el examen de Estado, los propósitos en materia de cobertura y las posibilidades de financiación a través del Icetex para programas de pregrado.

El informe de la ODDR también alude a la Resolución 163 de 2011, derogada por la Resolución 754 de 2013, mediante la cual se priorizan las estrategias orientadas al acceso a programas de formación para el trabajo que centran su mirada en acciones de formación complementaria, que otorguen el certificado de aptitud ocupacional, a nivel operario o auxiliar 
que conlleven titulación y oferta en niveles técnico, técnico profesional o tecnológico. En materia de educación superior se fijaba un estímulo económico que permitía destinar hasta el 50 \% del beneficio para cubrir los gastos de matrícula en un programa académico al cual se encontrara inscrito.

Con ocasión de la firma del acuerdo de paz, así como de las necesidades de la población en proceso de reincorporación, se profiere la Política Nacional para la Reincorporación Social y Económica de exintegrantes de las FarcEP, recogida en el documento Conpes 3931 de 2018. Cabe destacar de este documento la consideración que se desarrolla en materia educativa como parte de la definición de la política que busca "garantizar la reincorporación integral de exintegrantes de las Farc-EP y sus familias a la vida civil, de acuerdo con sus intereses y en el marco del Acuerdo Final” (DNP, 2018).

En el eje "Condiciones para el acceso y la atención de los derechos fundamentales e integrales de exintegrantes de las Farc-EP y sus familias", del Conpes 3931, se plantean como acciones que harán posible este objetivo el acceso, la permanencia y la continuidad en la educación formal, la cual se orienta además por la promoción de los escenarios de educación básica, media, técnica, tecnológica y universitaria para identificar y promover una respuesta institucional a la validación y homologación de saberes y conocimientos previos de los integrantes del grupo, lo que constituye un desafío y un hallazgo frente a las experiencias vividas en materia de reintegración.

Dentro de este marco de acción se identifican tres apuestas que deben desarrollarse y que implican la concentración de esfuerzos importantes, como la adecuada caracterización e identificación de las necesidades de 
la población, la reducción de riesgos de deserción y las estrategias de acceso, permanencia y continuidad.

De esta manera, el documento fija la hoja de ruta que debe ser seguida, que determina temporalmente las actividades y los esfuerzos importantes a emprender en materia de educación básica y media. Sin embargo, para los efectos de este artículo, resulta importante señalar que en el segundo semestre de 2018 se estableció que se fijarían los lineamientos para fomentar el acceso y la permanencia de los exintegrantes Farc-EP y sus familias en las IES, a partir de la conformación de una mesa técnica dedicada a este asunto. Asimismo, se indica que estos "modelos deberán cumplir con las competencias y los estándares de calidad establecidos previamente por el Ministerio de Educación Nacional” (DNP, 2018, p. 115). En el mismo sentido, el Conpes 3931 señala que, a partir del primer semestre de 2019, la ARN apoyaría el acceso y permanencia de exintegrantes de las Farc-EP a los programas de educación superior, y en el segundo semestre de ese año se realizaría la coordinación con el CNR para brindar el apoyo orientado a la promoción de la continuidad del proceso educativo con miras al acceso a la educación superior. Asimismo, se estableció como necesidad transversal impulsar, gestionar y fomentar el desarrollo de competencias para la convivencia, la reconciliación y la paz que tengan como destinatarios a la ciudadanía en general, para alcanzar los objetivos del Acuerdo Final.

El desafío del cambio de gobierno y la ausencia de posturas institucionales claras generó una dificultad no solo en la implementación de algunas estrategias, sino también en el seguimiento adecuado de las existentes, ante la definición necesaria de aspectos neurálgicos del proceso que fueron redimensionados por el gobierno del presidente Iván Duque y que continúan pendientes de ser aclarados. 
Pese a lo anterior, el 24 de diciembre de 2019, se expidió la Resolución 4309 que entró en vigencia el 1 de enero de 2020 y fijó la Ruta de Reincorporación. Este instrumento regulatorio era necesario no solo para el desarrollo del Sistema Nacional de Reincorporación que había previsto el Conpes 3931 de 2018 sino que además era requerido como consecuencia de los lineamientos fijados por el PND 2018-2022. Esta Resolución establece las características y condiciones para acceder a los beneficios económicos y sociales previstos en el Decreto 899 de 2017. En su Artículo 4, explicita que la Ruta regulada se desarrolla en dos etapas: una denominada "temprana" y otra "a largo plazo", esta última conforme a las previsiones del Artículo 5, tiene seis componentes, entre los cuales se encuentra la educación.

Conforme a las normas de la Resolución citada, el componente de educación se entiende cumplido cuando el beneficiario haya alcanzado un nivel educativo a partir de quinto de primaria y hasta los niveles técnico profesional o tecnológico, o cuando haya participado en programas de formación para el trabajo y el desarrollo humano con una intensidad mínima de 800 horas o cuente con certificados por competencias.

De acuerdo con la Cartilla de Reincorporación Social y Económica divulgada por la ARN (2020), los modelos creados para el acceso a la educación básica primaria y secundaria, educación media, técnica y tecnológica plantean una flexibilidad necesaria para evitar escenarios de deserción mediante tres propuestas: "Arando la educación”, "Educación y formación" y "Maestro itinerante". Sin embargo, en párrafo seguido se señala de manera precisa: “Además, se gestionarán los mecanismos para facilitar el ingreso a programas de educación superior con instituciones avaladas por el Ministerio de Educación Nacional” (ARN, 2020b, p. 6). 
Con este somero panorama es posible identificar los distintos escenarios previstos en la normatividad que aluden a la educación en los procesos de reintegración y reincorporación. Sin embargo, es clara la apuesta prioritaria en relación con el papel estratégico y articulado que viene cumpliendo la educación técnica y tecnológica con los fines del proceso, en contraste con la formulación de estrategias poco claras en relación con la educación superior universitaria. Si bien una aproximación a esta puede estar orientada a la financiación de la matrícula, no puede agotarse en esta dado que involucra aspectos mucho más complejos.

\section{Barreras e iniciativas de abordaje en la relación entre la universidad y la población en proceso de reintegración y reincorporación}

En la interacción de los desmovilizados con la universidad surgen algunas barreras, además de las enunciadas, que ponen en evidencia los importantes desafíos que deben abordarse en esa relación. El riesgo a ser invisibilizado constituye tal vez una de las principales barreras, especialmente en los primeros años de carrera. La tendencia a concebir a la población de excombatientes como fuente de información para la elaboración de ponencias y artículos de divulgación científica o papers los asume como objeto de estudio y pasa por alto su carácter deliberante y propositivo en la generación de nuevo conocimiento.

Este cambio de paradigma supone una apuesta por la generación de condiciones de acceso y permanencia en la educación superior en contextos universitarios, tal como lo señala Pedro Medellín, director nacional de la ESAP, en el reporte de prensa del año 2019 con ocasión del plan de becas otorgado por su institución, una apuesta orientada a la gestión del conocimiento y el desarrollo de los territorios. 
Nosotros tenemos una experiencia y gran presencia como formadores en administración pública. Ellos, los cien beneficiarios de estos cupos gratis, son conocedores de la geografía y la dinámica social de estos territorios. Así que se trata también de trabajar juntos, sumar capacidades y conocimientos para que cada reincorporado se convierta en un eje de transformación y de construcción de nación en los territorios, en especial los más golpeados por el conflicto. (ESAP, 2019)

De otro lado, los requisitos de admisión basados en la competencia igualitaria formal constituyen desafíos para las IES que adelantan iniciativas en este sentido. En Colombia las acciones afirmativas para el acceso a las universidades no son un debate nuevo. La realidad es que la población desmovilizada ha estado fuera del sistema educativo formal e informal durante décadas. De ahí que surge la siguiente pregunta: ¿̇cómo nivelar a esta población con las otras personas que también persiguen un cupo en la universidad?

Cabe mencionar como un ejemplo al respecto la oferta de la Universidad Industrial de Santander (UIS) dirigida a la población excombatiente. Esta universidad pública prevé un proceso de admisión especial para aspirantes desmovilizados de manera individual o en forma colectiva en procesos de paz, en el Acuerdo n. ${ }^{\circ} 282$ del 7 de noviembre de 2017. Esto implica la reserva de un cupo por carrera para "bachilleres que han dejado las armas y estén catalogados como desmovilizados de grupos guerrilleros, paramilitares, al margen de la ley, de manera individual o de forma colectiva en el marco de un proceso de paz dirigido por el Gobierno Nacional" (UIS, Acuerdo 282 de 2017, art. 6).

Los excombatientes que deseen postularse a ese cupo por carrera deberán cumplir con el requisito de haber presentado el examen de Estado de 
educación media, la prueba Saber 11, con una vigencia no mayor a cinco años, sin que se exija obtener un puntaje determinado.

Indiscutiblemente, la exigencia de contar con un título de bachiller es razonable, pues esa es la certificación que asegura conocimientos generales necesarios para el estudio de una carrera universitaria. De modo que una vez se da la reinserción, quienes no cuenten con dicho título, deben comenzar con el proceso para obtenerlo y luego darle continuidad al ciclo educativo en el siguiente nivel de enseñanza.

Otra estrategia para superar estas barreras en el ingreso a la educación superior es la firma de convenios entre las universidades y la ARN, como ocurrió con la Universidad Surcolombiana, que permitió a tres exjefes guerrilleros cursar con gratuidad la Maestría en Conflicto, Territorio y Cultura.

Sin embargo, requisitos como el dominio de una segunda lengua, la presentación de entrevista y otros filtros de admisión, adicionales al título de bachiller, dificultan el ingreso de desmovilizados a las universidades y, por tanto, constituyen aspectos de priorización para el diseño de las propuestas.

En contraste con lo anterior, una vez han ingresado, el siguiente reto de los excombatientes es la permanencia. En ese proceso de vida universitaria es esencial concebir al nuevo estudiante como una persona que no es una armadura vacía de experiencias y conocimientos, sino, por el contrario, un ser humano que conserva y ha acumulado una serie de experiencias válidas que nutren el conocimiento que se construye en la universidad, por tanto, además de identificar su alteridad es imperativo brindar las condiciones para reconocer sus conocimientos previos, así como promover la adaptación al entorno universitario. La experiencia del programa Ser Pilo Paga da cuenta de la necesidad de trabajar en frentes adicionales 
ligados a la generación de espacios de convivencia a la par de estrategias de financiación.

Retomando la referencia a la armadura vacía, es importante, además, continuar avanzando de manera decidida en la comprensión de que cada una de las funciones que los excombatientes ejercieron en el grupo armado cultivó en ellos un saber particular, una pericia y una experticia que no puede ser desaprovechada. En este sentido, se destacan iniciativas como la de la Universidad Nacional de Colombia, que propuso validar los saberes de quienes ejercieron por años como enfermeros de los campamentos, directores de comunicaciones, encargados de prensa, cocineros, escritores de comunicados, etc. De modo que los excombatientes pudieran sentirse parte de la comunidad universitaria y, con ello, integrantes de la sociedad civil, pues su aporte y su talento son valorados y reconocidos, en un ejercicio que reafirma su ciudadanía.

Por lo tanto, como expone Pachón (2017, p. 298), las barreras que pueden advertirse en el acceso y permanencia de los excombatientes en la educación superior podrían agruparse en: 1) barreras de acceso, 2) barreras de permanencia y 3) barreras de graduación. Como sostiene el autor:

Entre los elementos que conforman las barreras de acceso a la educación superior el MEN identificó: 1) estigmatización social de esta población por parte de la sociedad en general y de la comunidad universitaria en particular; 2) deficiencias en competencias generales y específicas que faciliten el acceso a la educación superior; y 3) insuficiencia de propuestas académicas relacionadas con un eventual escenario de posconflicto y reconciliación que promuevan el acceso de la población al sistema de educación superior. Para superar estas barreras, el MEN trazó una serie de estrategias que van desde la promoción de escenarios de diálogo entre las instituciones del Estado y las IES a fin de incentivar el 
acceso de esta población, hasta la conformación de grupos, semilleros, observatorios y grupos de investigación que aborden esta temática.

En cuanto a los elementos que integran las barreras de permanencia en la educación superior, el MEN identificó las siguientes: 1) dificultades de adaptación a la vida universitaria y a los espacios de sociabilidad con la comunidad académica, y 2) falta de capacitación de docentes y recursos pedagógicos adicionales. Aquí las estrategias propuestas por el MEN se orientaron a promover procesos de formación a la comunidad académica - directivos, docentes, estudiantes y personal administrativo- en temas de posconflicto y reconciliación, así como el acompañamiento académico y psicosocial al desmovilizado por parte del Estado y de las IES.

Por último, la barrera de graduación en educación superior expone la dificultad de inserción en el mercado laboral del desmovilizado; aquí, la estrategia se centraliza en el sector productivo del país y la agencia interinstitucional para financiar proyectos productivos. (Pachón, 2017, p. 298; énfasis añadido)

El Centro de Estudios sobre Desarrollo Económico (CEDE) de la Universidad de los Andes sugiere que existe un optimismo de la población en proceso de reincorporación en relación con la reconciliación, el futuro y el respeto hacia ellos mismos (Arjona et ál., 2020), no obstante, el contexto de discriminación resulta relevante en el diseño de las políticas públicas en todos los órdenes de impacto. Según los hallazgos del estudio realizado, las personas que cuentan, por ejemplo, con más años de educación tienen más saberes previos, quienes viven con un mayor número de personas en el hogar y tienen bajas condiciones de salud perciben mayor discriminación, así como los exintegrantes que viven en zonas urbanas y han participado en actividades de reconciliación. En este sentido, el estudio señala: 
Primero, es clave investigar si la discriminación que siente la población con mayor educación y saberes previos, por ejemplo, se asocia con discriminación por su pasado como exintegrante o por su experiencia laboral y/o adquisición de capital humano. Segundo, es importante focalizar [el] esfuerzo en el ámbito urbano, donde los exintegrantes suelen reportar una mayor sensación de experimentar discriminación. En general, es esencial entender si la percepción de discriminación tiene que ver con barreras no relacionadas propiamente con la identidad de la persona como exintegrante de las Farc-Ep, para identificar en dónde deben focalizarse dichos esfuerzos de política pública. (CEDE, 2020, p. 20)

Asimismo, frente a la inclusión ${ }^{3}$ como componente necesario en los procesos que impulsa la educación superior, se identifica la necesidad de gestionar esfuerzos orientados a la prevención de la discriminación y la estigmatización por parte de la población civil, que no se limiten a las estrategias de interacción física, sino que promuevan el reconocimiento de la persona reincorporada como un interlocutor válido, dado que esto resulta esencial dentro de la dimensión universalizante del entorno universitario (CEDE, 2020, p. 29).

Tal como lo señala el estudio del CEDE, las personas en proceso de reincorporación suelen estar prevenidas con el hecho de revelar que pertenecieron a las Farc-EP, lo que puede explicarse por la ausencia de un trabajo sostenido con la población civil que permita generar confianza en las acciones específicas que se desarrollan en el proceso, así como sobre el

\footnotetext{
3 Este concepto es abordado en el documento explicativo del Acuerdo por lo superior 2034. Propuesta de política pública para la excelencia de la educación superior en Colombia en el escenario de la paz, donde se indica que la inclusión se entiende como: "la capacidad de potenciar y valorar la diversidad, promover el respeto a ser diferente y garantizar la participación de la comunidad en una estructura intercultural de los procesos educativos. La educación inclusiva es una de las estrategias para la inclusión social" (CESU, 2014, p. 11).
} 
compromiso que los reincorporados han asumido en este (CEDE, 2020, p. 29).

Los aspectos mencionados evidencian que las universidades deben superar importantes escollos e identificar las lecciones aprendidas a la fecha, para que el acceso de las personas reincorporadas no se reduzca a una mera estadística, sino que constituya un componente transformador altamente contributivo en la reconstrucción del tejido social.

\section{Retos y desafíos de la educación superior para personas en proceso de reincorporación}

La investigación que dio lugar a este artículo enfrentó varios retos durante el proceso de recolección de información debido al cambio de gobierno y a las revelaciones que un proceso en marcha implica para los involucrados. Por tanto, se recogen en este acápite los principales hallazgos cualitativos obtenidos de fuentes oficiales, tales como el MEN, el Consejo Nacional de Acreditación (CNA) y la (ARN). Igualmente, se incluyen los hallazgos obtenidos de la consulta de páginas oficiales de universidades, tanto del sector público como privado, específicamente de sus bases de datos y páginas web, así como la divulgación de los proyectos en marcha publicados en distintos medios de comunicación.

La reforma constante de las propuestas, las realidades de contexto, las políticas institucionales, así como la novedad de algunas de las iniciativas emprendidas, generan una dispersión de información y un monitoreo que no ha sido unificado en función de todas las aristas que conforman el proceso. En relación con el monitoreo de los planes en materia de educación superior, se percibe una ausencia de seguimiento riguroso, dado que se concentran los esfuerzos en la oferta de educación básica, media, técnica y tecnológica profesional, lo que se explica no solo porque son los 
primeros pasos de la Ruta de Reincorporación, sino porque además, como se ha indicado, tienen una incidencia directa en las alternativas de subsistencia y desarrollo de proyectos productivos que convocan los esfuerzos e intereses más apremiantes.

En efecto, una de las iniciativas más extendida y que cuenta con una amplia documentación es la propuesta que viene desarrollándose desde el año 2008 y concentra los esfuerzos en la construcción de un Modelo de Educación y Formación para la Reintegración (MEFR), que ha contado con tres fases de implementación. Los componentes de este Modelo: 1) Ruta de exploración del talento; 2) La escuela como escenario de convivencia y reconciliación; 3) Formación por competencias; y 4) Formación por competencias para la construcción de un proyecto de vida afectiva, productiva y ciudadana (ACR, 2015, p. 10).

Esta iniciativa se articula con el modelo educativo colombiano, que apunta en un primer momento a los ciclos propedéuticos orientados a la puesta en marcha del modelo de formación para la reintegración, los ciclos de formación básica y media y la formación técnica y tecnológica en el SENA (ACR, 2015, p. 111).

Pese a lo anterior, en relación con las propuestas que involucran a la educación superior no existen bases documentales claras y se identifican algunos desafíos que no solo constituyen presupuestos que deben ser tenidos en cuenta por las IES en su oferta, sino que también deben ser revisados por el gobierno nacional cuando se establezca la política de calidad para estas entidades. De manera que las propuestas regulatorias involucren estos desafíos y permitan medir y evaluar de forma alterna pero funcional las propuestas que las IES formulen para responder a las necesidades y contextos de la población en proceso de reincorporación (García y Castellanos, 2020). 
Por consiguiente, resulta prudente revisar algunas de las reflexiones que se identifican en el marco de la oferta de programas, así como algunas de las regulaciones y directrices del MEN.

\section{Criterios de oferta de programas}

Los documentos consultados dan cuenta de la necesidad de trabajar en aspectos como:

- Dados los enfoques que se plantean en la Política Nacional para la Reincorporación Social y Económica (PNRSE), es necesaria la creación de programas que evidencien modelos de educación inclusiva que superen los roles de género tradicionales.

- Estructurar modelos educativos flexibles que permitan la compatibilidad con el desarrollo de actividades económicas.

- Avanzar en el desarrollo de mecanismos que se orienten a la validación de saberes previos y homologaciones para quienes ya habían cursado algunos ciclos de formación universitaria, con marcado acento en lo técnico y lo tecnológico.

- Ampliación de la cobertura con mecanismos de admisión basados en criterios de igualdad y equidad, así como el diseño de estrategias que velen por la continuidad en el proceso y disminuyan la deserción.

- Estrategias de financiación.

- Promover la eliminación de las barreras de percepción y estereotipos.

- Superar la visión del reincorporado/reinsertado como fuente de información u objeto de estudio para asumirlos como parte del proceso de generación de nuevo conocimiento. 


\section{Lineamientos del MEN en el contexto del posconflicto}

La investigación permitió verificar la formulación de políticas orientadoras para la toma de decisiones, sustentadas en la expedición de una serie de documentos y normatividad que se concretan en un entramado de instrumentos que han sido ampliados por marcos regulatorios. Se destacan el Acuerdo por lo superior 20344, el Plan Decenal de Educación 2016-2026, el documento Conpes 3931 de 2018, el PND 2018-2022 y el reciente Acuerdo 02 de 2020, por medio del cual se actualiza el modelo de acreditación de alta calidad.

En todos estos documentos, así como en aquellos que circulan en las páginas institucionales, se insiste en los importantes desafíos que afronta la educación superior en temas como cobertura, deserción, construcción curricular y mejoramiento de la calidad5.

\footnotetext{
${ }^{4}$ Este Acuerdo se formuló con el objetivo de identificar la expectativa nacional en materia de educación superior luego de realizar un recaudo de información en las distintas zonas del país, así como recoger la opinión de expertos tanto nacionales como extranjeros. Los insumos recaudados permitieron que el Consejo Nacional de Educación Superior (CESU) formulara la propuesta de política pública para ser ejecutada en los próximos veinte años.

${ }^{5}$ Como problemas nodales de la educación inclusiva, se abordan las cuestiones de acceso, permanencia y graduación, donde se identifican problemas como: la ubicación geográfica de ciertas poblaciones, las limitaciones tecnológicas y de infraestructura que generan dificultades para acceder a la educación superior. También se encuentran problemas de orden público, violencia social y desplazamiento; un elevado índice de pobreza e indigencia; carencias en los procesos de articulación de la educación media con la educación superior, que no toman en cuenta las necesidades pedagógicas y características especiales de ciertas poblaciones; insuficiencia de propuestas académicas relacionadas con un eventual escenario de posconflicto; una reducida oferta de créditos educativos; la migración de los jóvenes que viven en zonas rurales hacia los centros urbanos; barreras arquitectónicas y urbanísticas en las IES; apoyos profesionales insuficientes para orientar a estudiantes que han ingresado con baja preparación académica o que presentan discapacidad o talentos excepcionales; falta de capacitación especial a los profesores y escasez de recursos pedagógicos; dificultades de adaptación a la vida universitaria por situaciones de discriminación; limitados programas de bienestar universitario; prácticas pedagógicas que no tienen en cuenta las características de los estudiantes y el contexto; rigidez en los procesos curriculares; alta repitencia; acompañamiento académico y metodológico insuficientes; y poca flexibilidad en requisitos de graduación o elevado costo de los derechos de grado.
}

Revista IUSTA

ISSN: 1900-0448 | e-ISSN: 2500-5286 | DOI: https://doi.org/10.15332/25005286

N. 055 | julio-diciembre del 2021 
No obstante, estos aspectos adquieren matices importantes en el marco de un escenario de posconflicto y no se recogen plenamente como criterios diferenciadores en las mediciones institucionales de la calidad de la educación superior. Esto se corrobora en el hecho de que no existen documentos oficiales que hayan sido socializados con la comunidad académica universitaria que permitan identificar desde la política de aseguramiento de la calidad y en el marco de la autonomía universitaria, cuáles podrían ser los criterios que acogen las realidades del posconflicto y podrían ser valorados con flexibilidad o bajo otras metodologías de medición.

Claramente se advierte que la información en torno a programas y condiciones de admisión y permanencia específicamente diseñadas para la población es escasa, cuestión que también reconoce la población beneficiaria, dado que a la fecha no existe información relevante sobre los procedimientos para acceder a la educación superior.

Más allá de los esfuerzos de ciertas entidades, se evidencia que las iniciativas en esta materia no representan una oferta amplia y de fácil acceso. Como esfuerzos visibles, se encuentran las iniciativas abanderadas por instituciones como la Universidad Distrital Francisco José de Caldas en el mes de julio de 2019, las becas otorgadas por la ESAP, la Universidad Elite y la OEI, entre otras ${ }^{6}$.

\footnotetext{
6 "De esta forma, las IES del sector público y privado, como la Universidad de Cartagena, la Universidad de Córdoba, la Universidad de Nariño, la Universidad Nacional de Colombia, la Universidad Militar Nueva Granada, la Universidad Nacional Abierta y a Distancia (UNAD), la Universidad Pedagógica Nacional de Colombia, la Universidad de Antioquia, la Escuela Superior de Administración pública (ESAP), la Pontificia Universidad Javeriana, la Universidad de los Andes, la Universidad del Rosario, la Universidad Santo Tomás, la Universidad Externado de Colombia, la Institución Universitaria de Envigado, la Corporación Universitaria Remington (Uniremington) y la Corporación Universitaria Empresarial de Salamanca han abierto espacios académicos para incorporar población desmovilizada, ya sea a través del otorgamiento de cupos específicos, de la reducción en los requisitos de ingreso, de la
} 
A inicios del año 2020, algunos diarios nacionales reportaron el otorgamiento de un programa de becas para acceso a la educación superior, liderado por la ARN y la ESAP. En dicha publicación se mencionaba una población de 12059 personas que ya contaban con título de bachiller, así como la intención de 2454 excombatientes de las Farc-EP interesados en cursar una carrera universitaria (El Espectador, 2020).

En los reportes también se destacan las homologaciones logradas por quienes ya habían cursado algunos semestres de una carrera universitaria $\mathrm{y}$ algunas becas a las que tuvo acceso un número limitado de personas. Asimismo, se expresan las dificultades administrativas y académicas que han tenido que afrontar. Incluso en la Ruta de la Reintegración se indica que los excombatientes podrán acceder al beneficio económico que se contempla para el pago de matrícula en una IES, solo para quienes estén inscritos y hayan sido aceptados en un programa de educación superior, o se plantea que, por ejemplo, para acceder a las becas se debe contar con los resultados de la prueba Saber 11, lo que si bien es necesario, también implica importantes desafíos dada la necesidad de nivelar a la población y lograr unas competencias básicas que les permitan obtener resultados destacables, de cara, además, a garantizar su permanencia en el sistema educativo.

A la fecha de elaboración del presente artículo (2020), no se tiene claridad sobre las condiciones preferenciales en materia de cupos, requisitos de ingreso y oferta de programas de pregrado o posgrado que recojan la especificidad de estos posibles estudiantes, dado que se trata de esfuerzos puntuales provenientes de cada universidad. Tampoco se conocen los lineamientos del MEN en relación con el marco de propuestas que pueden

entrega de becas o de otros beneficios económicos tanto para el acceso como para el acompañamiento en procesos de permanencia" (Pachón, 2017, p. 296). 
realizarse y que serían compatibles con los estándares fijados por esta entidad en relación con la calidad, lo que pone de presente el diálogo necesario entre la autonomía universitaria y las condiciones de aseguramiento, con miras a coordinar los esfuerzos de manera coherente.

Como se ha evidenciado, la oferta educativa en el marco de la reintegración y la reincorporación se ha concentrado en cualificar el dominio de un oficio a través de una formación técnica, que ha logrado avanzar en los procesos de homologación de saberes de los excombatientes ${ }^{7}$. Sin embargo, la estadística en relación con la oferta en programas de educación superior, así como sobre las condiciones preferentes de acceso a programas de pregrado y posgrado, continúa siendo una incógnita con escasos resultados. De hecho, existe incertidumbre en relación con las propuestas concretas en este sentido. $\mathrm{Si}$ bien es clara la importancia de promover escenarios para garantizar la sostenibilidad económica, el ingreso como estudiantes a programas de pregrado o incluso de posgrado no ha sido una prioridad en la regulación, lo que impide analizar las posibles compatibilidades, así como valorar el impacto en el proceso.

Uno de los desafíos más importantes en la garantía al derecho a la educación superior de los excombatientes es la construcción de un currículo ampliamente participativo y con espacios de debate, como insumo para una educación de calidad (Velandia y Sánchez, 2020). No

\footnotetext{
7 Uno de los escenarios de homologación de saberes más notorios vincula al sector de la salud, tal como lo evidencia la nota periodística realizada por el diario El Tiempo (Rueda, 2020), donde se menciona el proceso dirigido a identificar los aprendizajes de los exguerrilleros y permitir su vinculación laboral. "En el marco del proceso de reincorporación se puso en marcha un proyecto auspiciado por la Cruz Roja Noruega, la Cruz Roja Colombiana y el Servicio Nacional de Aprendizaje (SENA) dirigido a validar los conocimientos que poseen algunos de los excombatientes en el campo de la salud. [...] 'Los 52 años de trayectoria en la guerra generaron muchos saberes en los exguerrilleros. Así iniciamos todo un proceso de homologación de saberes', explica Laura" (Rueda, 2020).
} 
obstante, la población en proceso de reintegración y reincorporación actualmente es considerada una fuente de información y objeto de estudio, lo que impide verlos como generadores de nuevo conocimiento, de manera que su participación en la fijación de estos contenidos no se reconoce como significativa. Si se une esta premisa con la excesiva concentración de las IES acreditadas en las ciudades, es posible identificar grandes retos en materia de participación y cobertura.

\section{Conclusión}

La función esencial que cumple la educación en los procesos de reincorporación es evidente, Joshua Mitrotti señala que a través de ella "las personas mejoran sus capacidades en términos de conocimiento y valores que promueven su desarrollo personal y social, con lo cual facilitan su acceso a oportunidades productivas, apoyando el desarrollo efectivo de su proyecto de vida en la legalidad" (ACR, 2015, p. 6). Dada la caracterización de la población, así como las necesidades que plantea el posconflicto, los avances en la materia reflejan importantes esfuerzos institucionales que tienen resultados destacados, recogidos en documentos institucionales cuya importancia no resulta posible minimizar.

No obstante, es claro que las necesidades de contexto, así como la factibilidad a corto plazo, ha llevado a que las propuestas gubernamentales le apuesten a la educación técnica y tecnológica de manera preferente, la cual si bien permite avanzar en la formación para el trabajo, no establece una hoja de ruta que garantice cumplir con la expectativa de la población en proceso de reincorporación de acceder a la educación superior, una aspiración que se muestra como una oportunidad esquiva y poco realizable. 
Las barreras que afrontan las personas en proceso de reintegración, que deben ser tenidas en cuenta por las IES en sus propuestas, se concentran en las condiciones de admisión, la homologación de saberes, la nivelación de conocimientos, los convenios, la financiación y la flexibilidad, lo que exige una gestión unificada, una priorización y un decidido apoyo desde las instituciones del Estado, para que las IES puedan formular propuestas en desarrollo de la autonomía universitaria en el marco de políticas coherentes y articuladas.

Asimismo, la modificación de los lineamientos en función de los gobiernos o administraciones de turno dificulta la ejecución de las iniciativas, lo que genera incertidumbre ante la ausencia de una postura clara frente a los retos del proceso. La importancia de una voluntad política firme y un respaldo decidido desde el sector central de la función pública pueden dotar de mayor confianza el proceso, no solo para la población directamente beneficiada sino para la sociedad civil en general, que muchas veces, a pesar de considerar necesario el acuerdo de paz, ve con alto escepticismo la contribución del gobierno central y de los exintegrantes de las Farc-EP al proceso, lo que profundiza la problemática de la discriminación y la estigmatización que impacta contextos específicos, como, por ejemplo, los escenarios universitarios.

Los reparos, críticas y reflexiones a documentos que fijan las políticas que determinan a la educación superior, tales como el Acuerdo por lo superior 2034, no han sido superados y abordados a la fecha con la profundidad requerida, lo cual incide negativamente en la formulación de los derroteros que orientan las propuestas para la población en proceso de reintegración. Si bien el Acuerdo mencionado fija unas metas, se requieren compromisos reales en materia de financiación de la educación superior para alcanzar 
los objetivos de admisión, permanencia y calidad, sin que en esto se agote el esfuerzo.

En el mismo sentido, la reflexión debe permear la articulación que puede realizarse a nivel regional con las IES que cuentan con trayectoria y reconocimiento a nivel nacional y que suelen ubicarse en las ciudades. Como señala Pachón (2017), las IES que el autor denomina de élite suelen recrear el discurso de la paz y generar desde el centro la política en este sentido, pero las IES gregarias que en su mayoría se encuentran en escenarios regionales o locales y recogen además a las instituciones de carácter técnico y tecnológico, son las que resultan encargadas de implementar y ejecutar la política, lo que evidencia la necesidad de repensar el modelo y generar espacios de articulación efectivos (Pachón, 2017, p. 302).

En este contexto sigue vigente la pregunta de Pachón (2017): ¿̇será que únicamente la inclusión que deben realizar las IES está destinada para aquellas que ofertan programas técnicos o tecnológicos, o aquellas que aún no cuentan con un amplio reconocimiento o estatus entre las IES, es decir, la IES gregarias? (p. 304). Consideramos que no, los hallazgos de estos años de implementación deberían ubicarnos en un terreno mucho más claro sobre el papel que pueden cumplir las universidades en el proceso de implementación del acuerdo de paz, las cuales a su vez deben recibir la confianza y el respaldo institucionales para emprender iniciativas innovadoras que se acompasen con los compromisos que les corresponde cumplir en materia de calidad y excelencia.

Igualmente, se destaca la importancia de reconocer la alteridad de las personas reincorporadas, lo que constituye una deuda en los escenarios educativos universitarios. Reconocerlos como sujetos activos que contribuyan al cumplimiento de la misión atribuida a las universidades es 
esencial, no basta con asumirlos como receptores de las políticas y estadísticas que se generan como evidencia del éxito de estrategias pensadas para ellos y no con ellos.

Así las cosas, la invitación que surge a partir de esta pesquisa se orienta a insistir en la importancia de que la propuesta formativa en materia educativa para la población en proceso de reincorporación gire su mirada hacia la universidad, para que esta, dentro de su autonomía, contribuya al reconocimiento de la alteridad de quienes han asumido el compromiso de vincularse a la institucionalidad, pero no como el personaje citado al inicio al inicio de estas páginas, un Agilulfo dueño de una armadura vacía que acata aquello que le es dado y cumple el libreto de la reincorporación, sino como un ciudadano respetuoso de la norma, con la posibilidad real de participar en espacios que tienen como misión innata promover la reflexión de la realidad y el ejercicio de una ciudadanía crítica y propositiva, comprometida con la vigencia de un orden justo.

El avance en esta aspiración constituye un asunto de urgente gestión que implica, a su vez, la formulación de otras preguntas entre las cuales puede encontrarse el interrogante con el que cerramos nuestra reflexión: ¿cómo podría diseñarse un conjunto de principios y criterios de gobierno, a partir de las nociones de excelencia y calidad de la educación superior, que sirva como herramienta para la construcción de políticas gubernamentales que orienten la oferta de programas académicos de educación universitaria, con énfasis en la equidad para la población en proceso de reintegración y reincorporación?

\section{Referencias}

Aristizábal, J. F. (2019). La teoría pura del derecho y la exclusión de la sicología. Revista IUSTA, 5O(1), 121-143. https://doi.org/10.15332/1900-0448.2019.0050.05 
Agencia Colombiana para la Reintegración [ACR]. (2015). Tiempo de aprender. http://www.reincorporacion.gov.co/es/la-reintegracion/centro-dedocumentacion/Documentos/Revista\%20Tiempo\%20Para\%20Aprender.pdf

Agencia para la Reincorporación y la Normalización [ARN]. (s. f.) ¿Qué es la reintegración? http://www.reincorporacion.gov.co/es/lareintegracion/Paginas/quees.aspx

Agencia para la Reincorporación y la Normalización [ARN]. (s. f.) ¿Qué es la reincorporación? http://www.reincorporacion.gov.co/es/reincorporacion/

Agencia para la Reincorporación y la Normalización [ARN]. (2019, 24 de diciembre). Resolución 4309. Por la cual se establece la Ruta de Reincorporación.

Agencia para la Reincorporación y la Normalización [ARN]. (2020a). ARN en cifras corte febrero de 2020.

http://www.reincorporacion.gov.co/es/agencia/Documentos\%20de\%20ARN\%20e n\%20Cifras/ARN\%20en\%20Cifras\%20\%20corte\%20enero\%202020.pdf

Agencia para la Reincorporación y la Normalización [ARN]. (2020b). Cartilla Reincorporación Social y Económica.

Arjona, A., Fergusson, L., Garbiras, N., García, J., Hiller, T., Polo, L. y Weintraub, M. (2020). Actitudes de exintegrantes de las Farc-EP frente a la reincorporación. (Documentos CEDE, $n .^{\circ}$ 24). Universidad de los Andes. https://egob.uniandes.edu.co/images/books/DT/DT-75.pdf

Calvino, I. (2002). El caballero inexistente. Almagro.

Centro Nacional de Memoria Histórica [CNMH]. (2017). Hacia el fin del conflicto: experiencias de desarme, desmovilización y paso de excombatientes a la vida civil en Colombia. Resumen. Panamericana. https://www.jep.gov.co/Sala-dePrensa/Documents/hacia-el-fin-del-conflicto.pdf

Centro de Estudios sobre Desarrollo Económico [CEDE]. (2020). Actitudes de exintegrantes de las Farc-EP frente a la reincorporación. https://repositorio.uniandes.edu.co/bitstream/handle/1992/41136/dcede202024.pdf? sequence $=1$ 
Colombia. Ley 30 de 1992. Por la cual se organiza el servicio público de la educación superior. Diciembre 28 de 1992. D.O. núm. 40700.

http://www.secretariasenado.gov.co/senado/basedoc/ley_o030_1992.html

Consejo Nacional de Educación Superior [CESU]. (2014). Acuerdo por lo superior 2034.

Propuesta de política pública para la excelencia de la educación superior en Colombia en el escenario de la paz.

https://www.mineducacion.gov.co/1621/articles-

344500 archivopdf sintesi doc acuerdosuperior.pdf

Departamento Nacional de Planeación [DNP]. (2015). Plan Nacional de Desarrollo 20142018.

https://observatorioplanificacion.cepal.org/sites/default/files/plan/files/Colombia _Plan_Nacional_de Desarrollo_2014_2018.pdf

Departamento Nacional de Planeación [DNP]. (2018). Política nacional para la reincorporación social y económica de exintegrantes de las Farc-EP. Conpes 3931. Consejo Nacional de Política Económica y Social. http://www.reincorporacion.gov.co/es/documents/conpes finlal web.pdf

Departamento Nacional de Planeación [DNP]. (2019). Plan Nacional de Desarrollo "Pacto por Colombia, pacto por la equidad 2018-2022". https://www.dnp.gov.co/DNPN/Paginas/Plan-Nacional-de-Desarrollo.aspx

Redacción Educación. (12 de enero de 2020). La ARN habilitó nuevas becas para que excombatientes puedan acceder a la educación superior. El Espectador. https://www.elespectador.com/educacion/la-arn-habilito-nuevas-becas-para-queexcombatientes-puedan-acceder-a-la-educacion-superior-article-899463/

Escuela Superior de Administración Pública [ESAP]. (2019, 19 de diciembre). ESAP otorgó 100 becas para excombatientes en proceso de reincorporación. Atención al ciudadano. https://www.esap.edu.co/portal/index.php/2019/12/19/esap-otorgo100-becas-para-excombatientes-en-proceso-de-reincorporacion/

García Lozano, L. F. y Castellanos Castillo, L. A. (2020). La sostenibilidad fiscal como limitante de la reparación administrativa de las víctimas de desplazamiento forzado. Via Inveniendi et Iudicandi, 15(2), 87-130.

https://doi.org/10.15332/19090528/6244

Revista IUSTA

ISSN: 1900-0448 | e-ISSN: 2500-5286 | DOI: https://doi.org/10.15332/25005286

N. ${ }^{\circ} 55$ | julio-diciembre del 2021 
Ministerio de Defensa Nacional (2003, 22 de enero). Decreto 128. Por el cual se reglamenta la Ley 418 de 1997, prorrogada y modificada por la Ley 548 de 1999 y la Ley 782 de 2002 en materia de reincorporación a la sociedad civil.

Ministerio de Educación Nacional [MEN]. (2017). Plan Decenal de Educación 20162026.

http://www.plandecenal.edu.co/cms/media/herramientas/PNDE\%20FINAL ISB N\%20web.pdf

Ministerio de Educación Nacional [MEN]. (2020, 1 de julio). Acuerdo 02. Por medio del cual se actualiza el modelo de acreditación de alta calidad.

Moreno Hurtado, E. y Álvarez Sierra, W. (2017). Presupuestos políticos y jurídicos para la reparación integral de las víctimas de desplazamiento forzado en Colombia a causa del conflicto armado. Via Inveniendi Et Iudicandi, 12(2), 117-147. https://doi.org/10.15332/s1909-0528.2017.0002.06

Navarrete, Z. (2013). La universidad como espacio de formación profesional y constructora de identidades. Universidades, 57, 5-16.

Nussbaum, M. (2010). Sin fines de lucro. Katz.

Pachón, W. (2017). Inclusión social de actores del conflicto armado colombiano: retos para la educación superior. Desafios, 3o(1), 279-308. https://doi.org/10.12804/revistas.urosario.edu.co/desafios/a.4917

Organización de Naciones Unidas [ONU]. (2019, 1 de octubre). Informe secretarial de la Misión de Verificación de las Naciones Unidas en Colombia. http://www.iri.edu.ar/wp-content/uploads/2019/12/bo-alatina-7o-onuinformesgcolombia.pdf

Ortega-Ruiz, L. G. y García Miranda, J. P. (2019). La verdad en la justicia transicional. Revista IUSTA, 1(50), 39-63. https://doi.org/10.15332/1900-0448.2019.0050.02

RevistaArcadia.com. (2018, 31 de julio). Así es la obra que Doris Salcedo está construyendo con las armas de las Farc. https://www.semana.com/arte/articulo/doris-salcedo-monumento-armas-de-lasfarc-acuerdos-de-paz/70319/\#

Revista IUSTA

ISSN: 1900-0448 | e-ISSN: 2500-5286 | DOI: https://doi.org/10.15332/25005286

N. ${ }^{0} 55$ | julio-diciembre del 2021 
RevistaArcadia.com. (2018, 11 de diciembre). Vea un clip del proceso de elaboración de 'Fragmentos'. https://www.semana.com/arte/articulo/vea-un-clip-del-proceso-deelaboracion-de-fragmentos/72314/

Rueda, C. (2020, 21 de julio). La médica de las Farc-EP que hoy vela por la salud del proceso de paz. El Tiempo. https://www.eltiempo.com/politica/proceso-de-paz/lamedica-que-hizo-11-anos-de-rural-en-las-farc-520668

Observatorio de Procesos de Desarme, Desmovilización y Reintegración [ODDR]. (2013). La educación superior y los procesos de reintegración en Colombia. Universidad Nacional de Colombia.

Universidad Industrial de Santander (UIS). Acuerdo 282 del 7 de noviembre de 2017.

Velandia Vega, J. y Sánchez Acevedo, M. E. (2020). ¿Cuál es la metodología para consolidar datos en una cultura de paz y reconciliación en Colombia? Via Inveniendi et Iudicandi, 15(2), 131-163. https://doi.org/10.15332/19090528/6245 